\title{
gos
}

\section{Dirac Equation and Quantum Relativistic Effects in a Single Trapped Ion}

\author{
L. Lamata, ${ }^{1}$ J. León, ${ }^{1}$ T. Schätz, ${ }^{2}$ and E. Solano ${ }^{3,4}$ \\ ${ }^{1}$ Instituto de Matemáticas y Física Fundamental, CSIC, Serrano 113-bis, 28006 Madrid, Spain \\ ${ }^{2}$ Max-Planck-Institut für Quantenoptik, Hans-Kopfermann-Strasse 1, D-85748 Garching, Germany \\ ${ }^{3}$ Physics Department, ASC, and CeNS, Ludwig-Maximilians-Universität, Theresienstrasse 37, 80333 Munich, Germany \\ ${ }^{4}$ Sección Física, Departamento de Ciencias, Pontificia Universidad Católica del Perú, Apartado Postal 1761, Lima, Peru
}

(Received 27 March 2007; published 22 June 2007)

\begin{abstract}
We present a method of simulating the Dirac equation in $3+1$ dimensions for a free spin- $1 / 2$ particle in a single trapped ion. The Dirac bispinor is represented by four ionic internal states, and position and momentum of the Dirac particle are associated with the respective ionic variables. We show also how to simulate the simplified $1+1$ case, requiring the manipulation of only two internal levels and one motional degree of freedom. Moreover, we study relevant quantum-relativistic effects, like the Zitterbewegung and Klein's paradox, the transition from massless to massive fermions, and the relativistic and nonrelativistic limits, via the tuning of controllable experimental parameters.
\end{abstract}

The search for a fully relativistic Schrödinger equation gave rise to the Klein-Gordon and Dirac equations. P. A. M. Dirac looked for a Lorentz-covariant wave equation that is linear in spatial and time derivatives, expecting that the interpretation of the square wave function as a probability density holds. As a result, he obtained a fully covariant wave equation for a spin-1/2 massive particle, which incorporated $a b$ initio the spin degree of freedom. It is known [1] that the Dirac formalism describes accurately the spectrum of the hydrogen atom and that it plays a central role in quantum field theory, where creation and annihilation of particles are allowed. However, the one-particle solutions of the Dirac equation in relativistic quantum mechanics predict some astonishing effects, like the Zitterbewegung and the Klein's paradox.

In recent years, a growing interest has appeared regarding simulations of relativistic effects in controllable physical systems. Some examples are the simulation of Unruh effect in trapped ions [2], the Zitterbewegung for massive fermions in solid state physics [3], and black-hole properties in the realm of Bose-Einstein condensates [4]. Moreover, the low-energy excitations of a nonrelativistic two-dimensional electron system in a single layer of graphite (graphene) are known to follow the Dirac-Weyl equations for massless relativistic particles [5,6]. On the other hand, the fresh dialog between quantum information and special relativity has raised important issues concerning the quantum information content of Dirac bispinors under Lorentz transformations [7].

In this Letter, we propose the simulation of the Dirac equation for a free spin- $1 / 2$ particle in a single trapped ion. We show how to implement realistic interactions on four ionic internal levels, coupled to the motional degrees of freedom, so as to reproduce this fundamental quantumrelativistic wave equation. We propose also the simulation of the Dirac equation in $1+1$ dimensions, requiring only the control of two internal levels and one motional degree of freedom. We study some quantum-relativistic effects, like the Zitterbewegung and the Klein's paradox, in terms of measurable observables. Moreover, we discuss the transition from massless to massive fermions, and from the relativistic to the nonrelativistic limit. Finally, we describe a possible experimental scenario.

We consider a single ion of mass $M$ inside a Paul trap with frequencies $\nu_{x}, \nu_{y}$, and $\nu_{z}$, where four metastable ionic internal states, $|a\rangle,|b\rangle,|c\rangle$, and $|d\rangle$, may be coupled pairwise to the center-of-mass (c.m.) motion in directions $x, y$, and $z$. We will make use of three standard interactions in trapped-ion technology, allowing for the coherent control of the vibronic dynamics [8]: first, a carrier interaction consisting of a coherent driving field acting resonantly on a pair of internal levels, while leaving untouched the motional degrees of freedom. It can be described effectively by the Hamiltonian $H_{\sigma}=\hbar \Omega\left(\sigma^{+} e^{i \phi}+\sigma^{-} e^{-i \phi}\right)$, where $\sigma^{+}$and $\sigma^{-}$are the raising and lowering ionic spin-1/2 operators, respectively, and $\Omega$ is the associated coupling strength. The phases and frequencies of the laser field could be adjusted so as to produce $H_{\sigma_{x}}=\hbar \Omega_{x} \sigma_{x}, H_{\sigma_{y}}=$ $\hbar \Omega_{y} \sigma_{y}$, and $H_{\sigma_{z}}=\hbar \Omega_{z} \sigma_{z}$, where $\sigma_{x}, \sigma_{y}$, and $\sigma_{z}$ are atomic Pauli operators in the conventional directions $x, y$, and $z$. Second, we consider a Jaynes-Cummings (JC) interaction, usually called red-sideband excitation, consisting of a laser field acting resonantly on two internal levels and one of the vibrational c.m. modes. Typically, a resonant JC coupling induces an excitation in the internal levels while producing a deexcitation of the motional harmonic oscillator, and vice versa. The resonant JC Hamiltonian can be written as $H_{r}=\hbar \eta \tilde{\Omega}\left(\sigma^{+} a e^{i \phi_{r}}+\sigma^{-} a^{\dagger} e^{-i \phi_{r}}\right)$, where $a$ and $a^{\dagger}$ are the annihilation and creation operators associated with a motional degree of freedom. $\eta=k \sqrt{\hbar / 2 M \nu}$ is the Lamb-Dicke parameter [8], where $k$ is the wave number of the driving field. Third, we consider an anti-JC (AJC) interaction, consisting of a JC-like coupling tuned 
to the blue motional sideband with Hamiltonian $H_{b}=$ $\hbar \eta \tilde{\Omega}\left(\sigma^{+} a^{\dagger} e^{i \phi_{b}}+\sigma^{-} a e^{-i \phi_{b}}\right)$. In this case, an internal level excitation accompanies an excitation in the considered motional degree of freedom, and vice versa.

All of these interactions could be applied simultaneously and addressed to different pairs of internal levels coupled to different c.m. modes. For example, it is possible to adjust field phases to implement a simultaneous blue- and red-sideband excitation scheme to form the Hamiltonian $H_{\sigma_{x}}^{p_{x}}=i \hbar \eta_{x} \tilde{\Omega}_{x} \sigma_{x}\left(a_{x}^{\dagger}-a_{x}\right)=2 \eta_{x} \Delta_{x} \tilde{\Omega}_{x} \sigma_{x} p_{x}$, with $i\left(a_{x}^{\dagger}-a_{x}\right) / 2=\Delta_{x} p_{x} / \hbar$. Here, $\Delta_{x}:=\sqrt{\hbar / 2 M \nu_{x}}$ is the spread in position along the $x$ axis of the zero-point wave function and $p_{x}$ the corresponding dimensioned momentum operator. The physics of $H_{\sigma_{x}}^{p_{x}}$ cannot be described anymore by Rabi oscillations. In turn, it yields a conditional displacement in the motion depending on the internal state, producing the so-called Schrödinger cat states $[9,10]$. By further manipulation of laser field directions and phases, we can also implement $H_{\sigma_{y}}^{p_{y}}=2 \eta_{y} \Delta_{y} \tilde{\Omega}_{y} \sigma_{y} p_{y}$ and $H_{\sigma_{x}}^{p_{z}}=2 \eta_{z} \Delta_{z} \tilde{\Omega}_{z} \sigma_{x} p_{z}$. This kind of interaction has already been produced in the lab, under resonant [11] and dispersive conditions [12].

We define the wave vector associated with the four ionic internal levels as

$$
|\Psi\rangle:=\Psi_{a}|a\rangle+\Psi_{b}|b\rangle+\Psi_{c}|c\rangle+\Psi_{d}|d\rangle=\left(\begin{array}{c}
\Psi_{a} \\
\Psi_{b} \\
\Psi_{c} \\
\Psi_{d}
\end{array}\right) .
$$

We may apply simultaneously different laser pulses, with proper directions and phases, $\eta \equiv \eta_{x}=\eta_{y}=\eta_{z}, \Delta \equiv$ $\Delta_{x}=\Delta_{y}=\Delta_{z}, \tilde{\Omega} \equiv \tilde{\Omega}_{x}=\tilde{\Omega}_{y}=\tilde{\Omega}_{z}, \Omega \equiv \Omega_{x}=\Omega_{y}=$ $\Omega_{z}$, to compose the following Hamiltonian acting on $|\Psi\rangle$,

$$
\begin{aligned}
H_{D}= & 2 \eta \Delta \tilde{\Omega}\left(\sigma_{x}^{a d}+\sigma_{x}^{b c}\right) p_{x}+2 \eta \Delta \tilde{\Omega}\left(\sigma_{y}^{a d}-\sigma_{y}^{b c}\right) p_{y}+ \\
& 2 \eta \Delta \tilde{\Omega}\left(\sigma_{x}^{a c}-\sigma_{x}^{b d}\right) p_{z}+\hbar \Omega\left(\sigma_{y}^{a c}+\sigma_{y}^{b d}\right) .
\end{aligned}
$$

We rewrite Eq. (2) in the suitable matrix form

$$
H_{D}=\left(\begin{array}{cc}
0 & 2 \eta \Delta \tilde{\Omega}(\vec{\sigma} \cdot \vec{p})-i \hbar \Omega \\
2 \eta \Delta \tilde{\Omega}(\vec{\sigma} \cdot \vec{p})+i \hbar \Omega & 0
\end{array}\right),
$$

where each entry represents a $2 \times 2$ matrix. The associated Schrödinger equation, $H_{D}|\Psi\rangle=i \hbar \partial|\Psi\rangle / \partial t$, performs the same dynamics as the Dirac equation in $3+1$ dimensions for a free spin- $1 / 2$ particle, where $|\Psi\rangle$ represents the fourcomponent Dirac bispinor. This is easily seen if we express the Dirac equation

$$
i \hbar \frac{\partial \psi}{\partial t}=\mathcal{H}_{D} \psi=\left(c \vec{\alpha} \cdot \vec{p}+\beta m c^{2}\right) \psi
$$

in its "supersymmetric" representation [1]

$$
\mathcal{H}_{D}=\left(\begin{array}{cc}
0 & c(\vec{\sigma} \cdot \vec{p})-i m c^{2} \\
c(\vec{\sigma} \cdot \vec{p})+i m c^{2} & 0
\end{array}\right) .
$$

Here, the $4 \times 4$ matrix $\vec{\alpha}:=\left(\alpha_{x}, \alpha_{y}, \alpha_{z}\right)=\operatorname{off}-\operatorname{diag}(\vec{\sigma}, \vec{\sigma})$ is the velocity operator, $\beta:=$ off-diag $\left(-i \mathbb{1}_{2}, i \mathbb{1}_{2}\right)$, and

$$
c:=2 \eta \Delta \tilde{\Omega}, \quad m c^{2}:=\hbar \Omega,
$$

are the speed of light and the electron rest energy, respectively. The notorious analogy between Eqs. (3) and (5) shows that the quantum-relativistic evolution of a spin- $1 / 2$ particle can be fully reproduced in a tabletop ion-trap experiment, allowing the study of otherwise inaccessible physical regimes and effects, as shown below.

In the Dirac formalism, the spin- $1 / 2$ degree of freedom is incorporated $a b$ initio. Moreover, the Dirac bispinor in Eq. (1) is built by components associated with positive and negative energies, $E_{\bar{D}}^{ \pm}= \pm \sqrt{p^{2} c^{2}+m^{2} c^{4}}$. This description is the source of diverse controversial predictions, as the Zitterbewegung and the Klein's paradox.

The Zitterbewegung is a known quantum-relativistic effect consisting of a helicoidal motion of a free Dirac particle, a natural consequence of the noncommutativity of its velocity operator components, $c \alpha_{i}$, with $i=x, y, z$. It can be proved straightforwardly [1] that the time evolution of the position operator $\vec{r}=(x, y, z)$ in the Heisenberg picture, following $d \vec{r} / d t=\left[\vec{r}, H_{D}\right] / i \hbar$, reads

$$
\begin{aligned}
\vec{r}(t)= & \vec{r}(0)+\frac{4 \eta^{2} \Delta^{2} \tilde{\Omega}^{2} \vec{p}}{H_{D}} t+\left(\vec{\alpha}-\frac{2 \eta \Delta \tilde{\Omega} \vec{p}}{H_{D}}\right) \frac{i \hbar \eta \Delta \tilde{\Omega}}{H_{D}} \\
& \times\left(e^{2 i H_{D} t / \hbar}-1\right) .
\end{aligned}
$$

Here, the first two terms on the right-hand side account for the classical kinematics of a free particle, while the last oscillating term is responsible for a transversal "quivering" motion. If we consider a bispinor state with a peaked momentum around $\quad p_{0}, \quad\left|\Psi_{0}\right\rangle=|a\rangle \otimes \exp [-(p-$ $\left.\left.p_{0}\right)^{2} / 2 \sigma_{p}^{2}\right]$, the Zitterbewegung frequency associated with the measurable quantity $\langle\vec{r}(t)\rangle$ can be estimated as

$$
\omega_{\mathrm{ZB}} \approx 2\left|\bar{E}_{D}\right| / \hbar \equiv 2 \sqrt{4 \eta^{2} \Delta^{2} \tilde{\Omega}^{2} p_{0}^{2} / \hbar^{2}+\Omega^{2}},
$$

where $\bar{E}_{D} \equiv\left\langle H_{D}\right\rangle$ is the average energy. Similarly, we can estimate from Eq. (7) the Zitterbewegung amplitude associated with $\langle\vec{r}(t)\rangle$ as

$$
R_{\mathrm{ZB}}=\frac{\hbar}{2 m c}\left(\frac{m c^{2}}{E}\right)^{2}=\frac{\eta \hbar^{2} \tilde{\Omega} \Omega \Delta}{4 \eta^{2} \tilde{\Omega}^{2} \Delta^{2} p_{0}^{2}+\hbar^{2} \Omega^{2}},
$$

and $R_{\mathrm{ZB}} \approx \Delta$, if $\eta \tilde{\Omega} \sim \Omega$.

The standard explanation of this erratic motion for a free Dirac particle invokes the interference between the positive- and negative-energy components of the Dirac bispinor following the dynamics in Eqs. (3) and (5). The predicted values for a real electron, $\omega_{\mathrm{ZB}} \sim 10^{21} \mathrm{~Hz}$ and $R_{\mathrm{ZB}} \sim 10^{-3} \AA$, are out of experimental reach, the effect 
has never been observed, and its existence is even questioned by quantum field theory considerations. To simulate quantum-relativistic effects in other physical systems, like trapped ions or graphene, is not aimed at proving their existence, but at exploiting the differences and analogies in each field. Given the flexibility of trapped-ion systems, we will have access to a wide range of tunable experimental parameters [8], allowing for realistic and measurable $\omega_{\mathrm{ZB}} \sim 0-10^{6} \mathrm{~Hz}$ and $R_{\mathrm{ZB}} \sim 0-10^{3} \AA$, depending on the initial vibronic states. Because of our piecewise buildup of the $3+1$ Dirac Hamiltonian of Eq. (2), we can strongly reduce the experimental demands to study Dirac equations in $1+1$ and $2+1$ dimensions $[1,13]$. In those cases, the Clifford algebra that characterizes the Dirac matrices is satisfied by the anticommuting Pauli matrices, $\left\{\sigma_{i}, \sigma_{j}\right\}=$ $2 \delta_{i j}$, where $\{A, B\}:=A B+B A$, given that we only need 2(3) anticommuting matrices in the $1+1(2+1)$ case. Accordingly, the four components of the Dirac bispinor are conveniently reduced to only two.

We focus now on the 1+1-dimensional case, which could be reached by current experiments, keeping most striking results available. After a unitary transformation around the $x$ axis, transforming $\sigma_{y}$ into $\sigma_{z}$, the $1+1$ Dirac equation stemming from Eq. (2) can be cast into $i \hbar \partial\left|\Psi^{(1)}\right\rangle / \partial t=H_{D}^{(1)}\left|\Psi^{(1)}\right\rangle$ with

$$
H_{D}^{(1)}=2 \eta \Delta \tilde{\Omega} \sigma_{x} p_{x}+\hbar \Omega \sigma_{z},
$$

where the Dirac "spinor" $\left|\Psi^{(1)}\right\rangle=\Psi_{a}^{(1)}|a\rangle+\Psi_{b}^{(1)}|b\rangle$. Note that these two components are not associated with the spin- $1 / 2$ degree of freedom but are linear combinations of positive- and negative-energy solutions [13]. Our setup is now reduced to a simultaneous $\mathrm{JC}+\mathrm{AJC}$ interaction, $\sigma_{x} p_{x}$, and a Stark-shift term $\sigma_{z}$ acting on two internal levels. In Eq. (10), the use of $\sigma_{z}$ is for the sake of pedagogy, and returning to $\sigma_{y}$ will not affect the results.

In the nonrelativistic limit, $m c^{2} \gg p_{x} c$, or equivalently $\Omega \gg \eta \tilde{\Omega}$, we may identify the dispersive limit in Eq. (10), and derive the squeezing second-order Hamiltonian

$$
H_{\mathrm{eff}}^{(1)}=\frac{2 \eta^{2} \Delta^{2} \tilde{\Omega}^{2}}{\hbar \Omega} \sigma_{z} p_{x}^{2} \equiv \sigma_{z} \frac{p_{x}^{2}}{2 m},
$$

yielding the expected Schrödinger Hamiltonian associated with the classical kinetic energy of a free particle. In the relativistic limit, $m c^{2} \ll p_{x} c$, which includes $m=0$, the $1+1$ case reduces to $H_{D}^{(1)}=i \hbar \eta \tilde{\Omega} \sigma_{x}\left(a_{x}^{\dagger}-a_{x}\right)$, which produces Schrödinger cats, as commented above $[9,10]$.

For a massless particle, we can show that $d x / d t=$ $\left[x, H_{D}^{(1)}\right] / i \hbar=2 \eta \Delta \tilde{\Omega} \sigma_{x}$ and $d \sigma_{x} / d t=\left[\sigma_{x}, H_{D}^{(1)}\right] / i \hbar=$ 0 . In consequence, $\sigma_{x}$ is a constant of motion and the time evolution of the position operator, $x(t)=x(0)+$ $2 \eta \Delta \tilde{\Omega} \sigma_{x} t$, is classical and does not involve Zitterbewegung. On the other hand, for a massive particle, $d \sigma_{x} / d t=\left[\sigma_{x}, H_{D}\right] / i \hbar=-\Omega \sigma_{y}, d \sigma_{y} / d t \neq 0$, and so forth. The produced set of differential equations yields a Zitterbewegung oscillatory solution similar to Eq. (7), with the evident simplification via the replacements of $H_{D}$ by $H_{D}^{(1)}, \vec{p}$ by $p_{x}$, and $\vec{\alpha}$ by $\sigma_{x}$. It is noteworthy to mention that the phenomenon of mass acquisition, which could be done here in a continuous manner by raising the coupling strength $\Omega$, is related to the spontaneous symmetry breaking mechanism of the Higgs field.

At this stage, it is clear that the measurement of the expectation value of the position operator, $\langle x(t)\rangle$, as a function of the interaction time $t$ is of importance. A recent proposal for realizing fast measurements of motional quadratures [14] relies on the possibility of measuring the population of an ionic internal level, $P_{a}$, at short probemotion interaction times $\tau$ with high precision [15]. Given an initial vibronic state $\rho(0)=\left|+{ }_{\phi}\right\rangle\left\langle+_{\phi}\right| \otimes \rho_{m}$, where $\left| \pm_{\phi}\right\rangle=\left(|a\rangle \pm e^{i \phi}|b\rangle\right) / \sqrt{2}$ and $\rho_{m}$ describes an unknown motional density operator, we can make use of

$$
\left\langle Y_{\phi}\right\rangle=\left.\frac{d}{d \tau} P_{e}^{+} \phi(\tau)\right|_{\tau=0},
$$

where the generalized quadrature $Y_{\phi}=\left(a e^{-i \phi}-\right.$ $\left.a^{\dagger} e^{i \phi}\right) / 2 i$. Then, position and momentum operators are measured when choosing $\phi=-\pi / 2$ and $\phi=0$, respectively. To apply this technique, it is required a particular initial state of the internal states, so the measurement of $\langle x(t)\rangle$ would have to be done in two parts, one associated with each of the suitably projected states, $\left|+_{\phi}\right\rangle$ and $\left|-_{\phi}\right\rangle$.

We turn now to the possible simulation of Klein's paradox. In 1929, Klein noticed [16] the anomalous behavior of Dirac particles in regions where a high potential energy $V$ exists: $H_{V}^{(1)}=H_{D}^{(1)}+V \mathbb{1}_{2}$. When $V>2 m c^{2}$, negativeenergy electrons (components) may swallow $V$, acquiring positive energy and behaving as ordinary electrons, while leaving a hole in the Dirac sea. This stems from the fact that the relativistic energy related to $H_{V}^{(1)}$ may be recast into $(p c)^{2}=\left(E_{V}^{(1)}-V+m c^{2}\right)\left(E_{V}^{(1)}-V-m c^{2}\right)$, which is positive when either both factors are positive or negative. In the second case, the total energy $E_{V}^{(1)}<-m c^{2}+V$ can be larger than $m c^{2}$, as noticed by Klein. In this case an $e^{-}-$ $e^{+}$(electron-positron) pair could be created from $V$. The sudden raise of the constant potential $V(|a\rangle\langle a|+| b\rangle\langle b|)$, at a certain time $t=t_{0}$ after an evolution associated with $H_{D}^{(1)}$, could simulate this phenomenon. We suggest to produce potential $V$ with the required characteristics through a fast and homogeneous Stark shift in both internal levels. The natural way to detect Klein's paradox, assuming an initial positive-energy internal state $|a\rangle\left(p_{0}=0\right)$, is via measurement of nonzero population in the negativeenergy component $|b\rangle$.

Finally, in as much as massless chiral fermions in condensed matter, we could also produce a $1+1$ axial anomaly [17] by changing Eq. (10) into $H_{D}^{(1)}=c \sigma_{x} p_{x}+q E x$, where $x \propto\left(a+a^{\dagger}\right)$ is a motional displacement. 


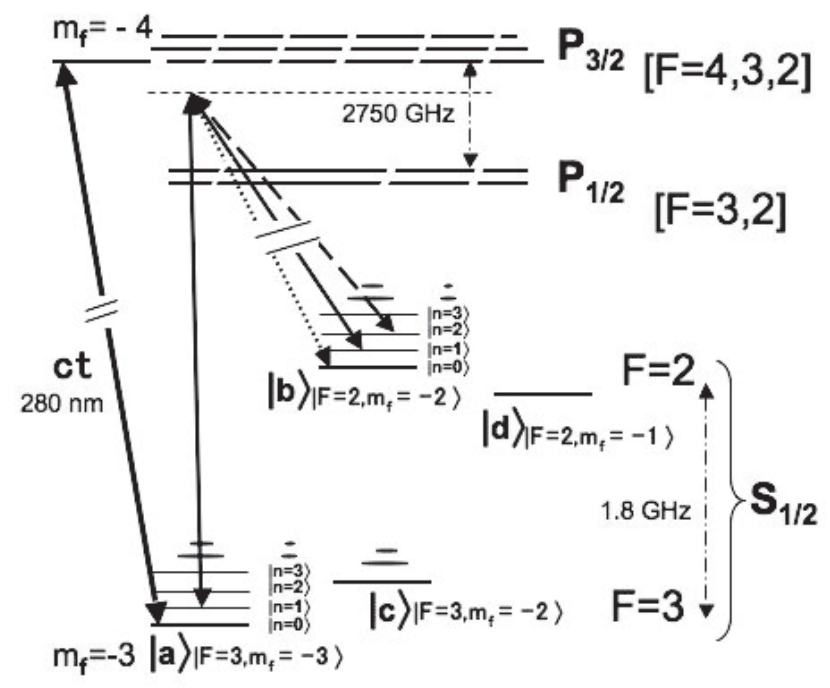

FIG. 1. Energy levels of a ${ }^{25} \mathrm{Mg}^{+}$ion confined in a trap $[22,23]$. Shown are the relevant types of two-photon Raman transitions between states $|a, n=1\rangle$ and $|b, n=0,1,2\rangle$ : red sideband (dotted line), carrier (solid line) and blue sideband (dashed line); see also the resonant transition for state sensitive detection. We subsumed excited levels of the $P_{1 / 2}$ and $P_{3 / 2}$ states.

The basic ingredients to implement the 1D Dirac dynamics of Eq. (10) with a single trapped ion are two independent electronic (internal) states coupled to a onedimensional motional degree of freedom. The required states could be composed by two ground-state hyperfine levels of an earth alkaline atomic ion, e.g., of ${ }^{25} \mathrm{Mg}^{+}$by $\left|F=3 ; m_{f}=-3\right\rangle$ and $\left|F=2 ; m_{f}=-2\right\rangle,|a\rangle$ and $|b\rangle$, respectively, as depicted in Fig. 1. A constant external magnetic field will define the quantization axis and lift the degeneracy of levels being potentially useful to provide additional states (like $|c\rangle$ and $|d\rangle$ in Fig. 1) necessary for higher dimensional simulations, see Eq. (2).

At the start, the ions will be laser cooled close to the motional ground state and optically pumped into state $|a\rangle$ [18]. One red or blue sideband and one carrier transition will be driven simultaneously $[11,19]$ to implement the desired dynamics of Eq. (5) via two-photon stimulated Raman transitions. To measure the ion position we rely on the mapping of motional information on the internal degrees of freedom $[14,15]$ and take advantage of the high fidelity of state sensitive detection realized by an additional laser beam, tuned to a cycling transition [20], coupling state $|a\rangle$ resonantly to the $P_{3 / 2}$ level. Considering the available laser intensities [21], all necessary Raman beams could be derived from a single laser source. We split the original beam and provide the necessary frequency offsets, phase control, and switching via multipassing through acousto-optical modulators [19]. The number of laser beams could be further reduced by electro-optical modu- lators to provide red and blue sidebands simultaneously $[11,12]$. To implement an overall shift in the potential (Stark shift of level $|a\rangle$ and $|b\rangle$ in Klein's paradox) without changing their mutual energy difference we chose the directions and polarizations of the Raman beams appropriately.

In conclusion, we have shown how to simulate the Dirac equation in $3+1$ dimensions for a free spin- $1 / 2$ particle, and quantum-relativistic effects like the Zitterbewegung and Klein's paradox, in a single trapped ion. We have studied the $1+1$ case, where experimental needs are minimal while keeping most striking predictions. We believe that these simulations open attractive avenues and a fruitful dialog between different scientific communities.

The authors thank D. Leibfried and J.I. Latorre for valuable comments. L.L. acknowledges support from MEC FPU Grant No. AP2003-0014. L. L. and J. L. were partially supported by the Spanish MEC FIS2005-05304 and CSIC 20045 0E 271 projects. T. S. acknowledges support by DFG, MPQ, and MPG. E. S. is grateful for the hospitality at CSIC (Madrid) and acknowledges support from EU EuroSQIP and DFG SFB 631 projects.

[1] B. Thaller, The Dirac Equation (Springer-Verlag, Berlin, 1992).

[2] P. M. Alsing, J. P. Dowling, and G. J. Milburn, Phys. Rev. Lett. 94, 220401 (2005).

[3] J. Schliemann, D. Loss, and R. M. Westervelt, Phys. Rev. Lett. 94, 206801 (2005).

[4] L. J. Garay et al., Phys. Rev. Lett. 85, 4643 (2000).

[5] J. Cserti and G. Dávid, Phys. Rev. B 74, 172305 (2006).

[6] M. I. Katsnelson, K. S. Novoselov, and A. K. Geim, Nature Phys. 2, 620 (2006).

[7] A. Peres and D. R. Terno, Rev. Mod. Phys. 76, 93 (2004).

[8] D. Leibfried et al., Rev. Mod. Phys. 75, 281 (2003).

[9] E. Solano, R. L. de Matos Filho, and N. Zagury, Phys. Rev. Lett. 87, 060402 (2001).

[10] E. Solano, G. S. Agarwal, and H. Walther, Phys. Rev. Lett. 90, 027903 (2003).

[11] P. C. Haljan et al., Phys. Rev. Lett. 94, 153602 (2005).

[12] C. A. Sackett et al., Nature (London) 404, 256 (2000).

[13] B. Thaller, arXiv:quant-ph/0409079.

[14] P. Lougovski, H. Walther, and E. Solano, Eur. Phys. J. D 38, 423 (2006).

[15] D. M. Meekhof et al., Phys. Rev. Lett. 76, 1796 (1996).

[16] O. Klein, Z. Phys. 53, 157 (1929).

[17] H. B. Nielsen and M. Ninomiya, Phys. Lett. 130B, 389 (1983).

[18] B. E. King et al., Phys. Rev. Lett. 81, 1525 (1998).

[19] D. J. Wineland et al., Phil. Trans. R. Soc. A 361, 1349 (2003).

[20] C. Monroe et al., Phys. Rev. Lett. 75, 4714 (1995).

[21] A. Friedenauer et al., Appl. Phys. B 84, 371 (2006).

[22] M. A. Rowe et al., Quantum Inf. Comput. 2, 257 (2002).

[23] F. Schmidt-Kaler et al., Appl. Phys. B 77, 789 (2003). 